(2) Open Access Full Text Article

REVIEW

\title{
Lapatinib for the treatment of breast cancer in the People's Republic of China
}

This article was published in the following Dove Press journal:

OncoTargets and Therapy

31 July 2014

Number of times this article has been viewed

\section{Hongjiang Wang}

Department of Breast Surgery, First Affiliated Hospital of Dalian Medical University, Dalian, People's Republic of China
Correspondence: Hongjiang Wang Department of Breast Surgery, First Affiliated Hospital of Dalian Medical University, 222 Zhongshan Road, Dalian, I I60 I I, People's Republic of China

Tel +86 4II 83635963 ext 2085 Fax +86 4II 83622844

Email hjwang@dlmedu.edu.cn
Abstract: Lapatinib is an oral, small-molecule, reversible inhibitor of both epidermal growth factor receptor and human epidermal growth factor receptor-2 (HER2) tyrosine kinases. In March 2007, the US Food and Drug Administration approved lapatinib for use in combination with capecitabine for the treatment of women with HER2-overexpressing, advanced or metastatic breast cancer. This review discusses the available information of lapatinib in Chinese breast cancer patients, focusing on its effectiveness and clinical application against advanced or metastatic breast cancer. In pivotal phase III trials, a combination of lapatinib and capecitabine significantly decreased the risk of disease progression compared to capecitabine alone in women with HER2-positive advanced or metastatic breast cancer. Other trials were used to evaluate lapatinib in combination with hormone therapy, in combination with trastuzumab, and as an adjunct to adjuvant therapy for early-stage disease. Preclinical data have revealed that lapatinib is active in trastuzumab-resistant cell lines as well as synergistic with trastuzumab. In clinical trials, lapatinib has not been associated with serious or symptomatic cardiotoxicity. Further, it can cross the blood-brain barrier and may therefore have a role in preventing cancer progression in the central nervous system. Thus, lapatinib warrants further evaluation in HER2-positive metastatic and early-stage breast cancer patients.

Keywords: lapatinib, HER2 positive, breast cancer, molecular targeting therapy

\section{Introduction}

Breast cancer is the most common cancer among women worldwide. About half of the breast cancer cases and $60 \%$ of the deaths are estimated to occur in economically developing countries. ${ }^{1}$ Approximately $16 \%-20 \%$ of women with breast cancer have advanced or metastatic breast cancer, while $50 \%$ of early-stage breast cancers ultimately develop into metastatic breast cancer. ${ }^{2}$ Advanced or metastatic breast cancer is defined as a clinical stage that corresponds to cancer stage III and IV, based on the tumor itself, on lymph node involvement, and on metastases. Approximately $15 \%-22 \%$ of breast cancers overexpress human epidermal growth factor receptor 2 (HER2), ${ }^{2,3}$ placing patients at increased risk for disease progression and death. ${ }^{3,4}$ Progress in the treatment of HER2-positive breast cancer has been achieved by targeting HER2 signaling pathways. Trastuzumab is a monoclonal antibody that binds the extracellular domain of the HER2 receptor and has been the basis of therapy for patients with HER2-positive metastatic breast cancer, although resistance ultimately develops. ${ }^{5,6}$

Lapatinib is an orally active, dual inhibitor of HER2 and epidermal growth factor receptor(EGFR), marketed by a pharmaceutical company in England, GlaxoSmithKline (GSK) in 2001. In March 2007, the US Food and Drug Administration (FDA) approved 
lapatinib for use in combination with capecitabine in the treatment of advanced HER2-amplified (HER2-positive) breast cancer. ${ }^{7}$ Like trastuzumab, lapatinib is indicated only for the treatment of HER2-positive breast cancer. The goal of this review is to summarize lapatinib's approved and investigational uses in the People's Republic of China.

\section{Mechanism of action of lapatinib}

Lapatinib is a 4-anilinoquinoline derivative. It potently but reversibly binds to the intracellular tyrosine kinase domains of EGFR and HER2, leading to inhibition of substrate phosphorylation. ${ }^{89}$ Lapatinib competes for the adenosine triphosphate (ATP)-binding site on the intracellular tyrosine kinase domains of both EGFR and HER2, thus preventing the use of ATP as a cofactor for phosphorylation of the tyrosine residue. ${ }^{10,11}$ This inhibition blocks downstream mitogen-activated protein kinase (MAPK), phosphoinositide 3-kinase (PI3K) proliferation, and survival signaling pathways, thereby rendering antineoplastic activity. ${ }^{12,13}$ In vitro and in vivo studies have demonstrated that lapatinib has an inhibitory effect on a variety of solid tumors. ${ }^{14-17}$ Depending on tumor type, lapatinib-treated cells may undergo either apoptosis or growth arrest.

Lapatinib can be administered orally and is distributed well to many organs. Although lapatinib uptake in a normal brain is $<10 \%$ of plasma due to active efflux transport at the brain-blood barrier, ${ }^{18,19}$ it has increased activity in disease affecting the central nervous system (CNS), possibly because the blood-tumor barrier is more permissive. ${ }^{20-22}$ This has been demonstrated in mice with HER2 overexpressing brain metastases, where treatment with lapatinib resulted in a significant decrease in tumor volume. This was attributed to a decrease in HER2 phosphorylation and cell proliferation. ${ }^{23}$ In clinical studies, lapatinib is found to provide modest but definite evidence of anti-tumor activity in patients with brain metastases from breast cancer. ${ }^{24,25}$

Lapatinib is highly potent with a $50 \%$ inhibitory concentration (IC50) of $<0.2 \mu \mathrm{m}$ for both EGFR and HER2. ${ }^{26}$ Its slow rate of dissociation from the intracellular tyrosine kinase domains of EGFR and HER2 (half-life $\geq 300$ minutes) correlates with a prolonged downregulation of receptor tyrosine phosphorylation in tumor cells. ${ }^{26}$ In addition, lapatinib effectively inhibits human tumor cell lines that overexpress EGFR or HER2, which indicates selectivity for cancer cells that overexpress these receptors. ${ }^{27}$ Further preclinical and clinical trials indicate that lapatinib is considerably less cardiotoxic than trastuzumab, ${ }^{28-31}$ which has been proposed to relate to off target cardioprotective effects of lapatinib. ${ }^{32}$ Alternatively, differences in drug pharmacokinetics and in the duration of ErbB2 blockade between lapatinib and trastuzumab, as a result of their distinct modalities, may also account for the difference in cardiotoxicity.

Basic research has been performed in the People's Republic of China on the mechanisms of action and drug resistance of lapatinib in the treatment of breast cancer. In vitro studies have shown that IC50 of lapatinib was less than $0.16 \mathrm{~mol} / \mathrm{L}$ in A431, HN5, BT474, N87, and CaLu-3 cell lines, and was about $3 \mathrm{~mol} / \mathrm{L}$ in MCF-7 and T47D cell lines. Compared to EGFR- or/and HER2-positive cell lines, breast cancer cells with low EGFR or/and HER2 expression had 25 times higher IC50. In addition, HN5 and BT474 breast cancer cell growth was completely inhibited in nude mice xenograft models after treatment of lapatinib $0.1 \mathrm{mg} / \mathrm{g}$, two times/day. ${ }^{14}$ Apoptosis increased 23 times in lapatinibtreated tumor cells with HER2-overexpression and lapatinib anti-tumor activity was not suppressed by EGF. ${ }^{9}$ In addition, lapatinib exhibited in vitro additive effects in combination with fluorouracil (the active metabolite of capecitabine) ${ }^{33}$ and demonstrated enhanced or synergistic effects in combination with trastuzumab. ${ }^{10}$ Lapatinib induced P27 expression, subsequently combined and inhibited CDK2 function, and therefore restored breast cancer cells to hormonal drug tamoxifen sensitivity. ${ }^{34}$ Konecny et a ${ }^{10}$ found that a combination of lapatinib and trastuzumab increased the treatment effect. The interaction between lapatinib and trastuzumab involves lapatinib-induced stabilization and accumulation of HER2 at the cell surface, which in turn facilitates immune-mediated, trastuzumab-dependent cytotoxicity. ${ }^{35}$

Li et al ${ }^{36}$ established a lapatinib-resistant breast cancer cell line rBT474, which was found to have changed HER2 and ER alpha signaling pathways. Compensatory activation of ER alpha pathway may be the cause of lapatinib resistance in HER2 (+)/ER $\alpha(+)$ breast cancer. PI3K/AKT suppression and MAPK activation may be responsible for the ER alpha compensatory activation. Zhao ${ }^{37}$ also found that lapatinib resistance in breast cancer may be associated with Wnt/Beta-catenin signaling pathways. Betacatenin knockdown by small interfering RNA (siRNA) significantly increased lapatinib-induced apoptosis in lapatinib-resistant breast cancer cells. Other studies indicated that apoptosis induced by lapatinib in HER2 overexpressing breast cancer cell line SKBR-3 is related to downregulation of p-AKT, and survivin..$^{38}$ Additional studies are warranted. 


\section{Application of lapatinib for the treatment of breast cancer in Western countries}

Studies have found that a variety of tumors overexpress HER2, including $15 \%-22 \%$ breast cancer, $25 \%-32 \%$ ovarian cancer, and $30 \%-35 \%$ lung adenocarcinoma. ${ }^{39}$ With the development of basic research and clinical trials of molecular targeted therapy in HER2 overexpressing breast cancer, lapatinib is becoming a new adjuvant therapeutic option. EGF103009 studies have shown that lapatinib is mainly used in combination with capecitabine for the treatment of patients with advanced or metastatic HER2-positive breast cancer. ${ }^{33}$ The pivotal phase III study EGF100151 demonstrated that the addition of lapatinib to capecitabine significantly decreased the risk of disease progression compared with capecitabine alone in women with HER2-positive advanced or metastatic breast cancer that had not responded to previous treatment with an anthracycline, a taxane, and trastuzumab. ${ }^{40}$ In this study, a total of 399 women were randomized to either lapatinib $1,250 \mathrm{mg} /$ day plus capecitabine $2,000 \mathrm{mg} / \mathrm{m}^{2}$ given on days 1-14 of a 21-day cycle (combination therapy) or capecitabine $2,500 \mathrm{mg} / \mathrm{m}^{2}$ on the same schedule (monotherapy). On the basis of data from the first 324 patients, the risk of disease progression was significantly reduced with combination therapy compared to monotherapy (hazard ratio [HR] $0.49,95 \%$ confidence interval [CI] $0.34-0.71, P<0.001)$. The median times to disease progression were 8.4 and 4.4 months, respectively. Cox regression analysis indicated that treatment assignment was the only significant factor affecting the time to disease progression.

A large clinical trial has also shown that lapatinib is beneficial in the treatment of HER2-positive early stage breast cancer. ${ }^{41}$ In this randomized, controlled, phase III clinical trial, Goss et $\mathrm{al}^{41}$ studied 3,147 early-stage breast cancer patients across 33 countries. Patients were randomly assigned (1:1) to receive daily lapatinib $(1,500 \mathrm{mg})$ or daily placebo for 12 months. After a median follow-up of 47.4 months (range 0.4-60.0) in the lapatinib group and 48.3 (range 0.7-61.3) in the placebo group, disease-free survival did not differ significantly between groups in the primary analysis. There was no significant difference in CNS recurrences and no evidence existed for a survival advantage with lapatinib treatment. However, disease-free survival was better in the lapatinib group for women with hormone-receptor-negative tumors and there was a suggestion of benefit for patients who received lapatinib within a year of diagnosis. Exploratory analysis was restricted to 2,490 patients with HER2-positive tumors which suggested improvement in disease-free survival with lapatinib. Thus, lapatinib may be an option for women with HER2-positive breast cancer who did not or cannot receive adjuvant trastuzumab.

\section{Clinical trials of lapatinib in the People's Republic of China}

Lapatinib has been approved by the FDA primarily based on EGF103009 randomized phase III clinical trials. The treatment of breast cancer in Asia is largely similar to that in Western countries, however, several distinct factors in Asia have an impact on clinical decision making, including cost considerations, agent availability due to regulatory approval, and ethnic differences. ${ }^{42,43}$

Han et $\mathrm{al}^{44}$ performed a randomized, non-blind, multicenter, phase III clinical trial in the People's Republic of China on HER2-positive advanced breast cancer patients, who had failed previous chemotherapy by anthracycline, taxane, and/or trastuzumab. The patients were treated with either a combination of lapatinib $(1,250 \mathrm{mg} / \mathrm{d})$ and capecitabine $\left(2,000 \mathrm{mg} / \mathrm{m}^{2}\right.$, $\mathrm{d} 1 \sim \mathrm{d} 14)$, or capecitabine $\left(2,500 \mathrm{mg} / \mathrm{m}^{2}, \mathrm{~d} 1 \sim \mathrm{d} 14\right)$ alone for 3 weeks. A combination of lapatinib and capecitabine reduced the risk of disease progression by $51 \%$, which indicates that lapatinib is an efficient and well tolerated treatment for HER2-positive advanced breast cancer. Xu et al ${ }^{45}$ also took a single-arm, open-label approach to study the efficacy and safety of lapatinib in a Chinese population. The patients had stage IIIB/IIIC-IV, HER2-positive advanced or metastatic breast cancer, and previously received single or combination treatment of anthracycline, taxane, and/or trastuzumab. All patients received lapatinib (1,250 mg once daily) and capecitabine $\left(2,000 \mathrm{mg} / \mathrm{m}^{2}\right.$ per day) on days 1 to 14 every 21 days. Twenty-three patients had partial response, seven patients were stable for at least 24 weeks, and zero patients showed complete response. These resulted in a clinical benefit rate of $57.7 \%$. The median progression-free survival was 6.34 months. The median time to response and duration of response were 4.07 months and 6.93 months, respectively. Thirteen (25.0\%) patients had disease progression with new lesions. These data confirmed that the combination of lapatinib and capecitabine is an effective and well-tolerated treatment option in a population of Chinese women with HER2-positive advanced or metastatic breast cancer who have had disease progression following other treatments. Interestingly, the clinical benefit rate of lapatinib in a Chinese population (57.7\%) is considerably higher than those in a Western population reported by Geyer et $\mathrm{al}^{140}$ and Cameron et $\mathrm{al}^{146}$ ( $27 \%$ and $29.3 \%$, respectively). The median progression-free survival of 6.34 months observed in the Chinese breast cancer patients compared favorably with 
the 6.2 months reported by Cameron et al. ${ }^{46}$ These results are consistent with previously reported improved responses of Asian ethnicity to EGFR inhibitors in other solid tumors. ${ }^{47,48}$ Based on these studies, lapatinib is currently approved for using in metastatic breast cancer treatment in the People's Republic of China.

Evaluation of lapatinib as a first-line adjuvant chemotherapy for HER2-positive early-stage breast cancer is ongoing. There are also many phase III clinical trials being performed in other countries. For example, EFG30001/ EGF104353 is the comparison of paclitaxel plus lapatinib and paclitaxel plus placebo; EFG30008 letrozole plus lapatinib and letrozole plus placebo; EFG104383 paclitaxel plus trastuzumab plus lapatinib and paclitaxel plus trastuzumab plus placebo et al.

\section{Clinical application of lapatinib in the People's Republic of China}

The purposes of metastatic breast cancer treatment are to extend survival time and improve quality of life. ${ }^{49}$

According to National Comprehensive Cancer Network Breast cancer clinical practice guidelines as well as the Chinese Anti-cancer Association Breast Cancer Committee, ${ }^{50}$ HER-2 positive metastatic breast cancer treatment options and guidelines are as follows: trastuzumab-based therapy as first-line choice, better combined with other chemotherapy drugs. Combined chemotherapies include paclitaxel or docetaxel, vinorelbine, platinum class, capecitabine, and gemcitabine.

For breast cancers positive for both hormone receptors and HER-2, trastuzumab can be combined with aromatase inhibitors such as anastrozole. If the tumor progressed after trastuzumab-based combination chemotherapy, trastuzumab can be continued or be substituted with other combination chemotherapies or a combination of lapatinib and capecitabine. ${ }^{51}$

A randomized clinical trial ${ }^{52}$ indicated that lapatinib and capecitabine combination is more effective than capecitabine alone in treatment of HER2-positive metastatic breast cancer. Compared to capecitabine alone, lapatinib and capecitabine combination significantly extended patients' progressionfree survival and median time to disease progression and reduced the incidence of brain metastases. The incidences of adverse reactions are similar between the two groups. A randomized phase III clinical trial, comparing treatment effects of paclitaxel plus lapatinib combination and paclitaxel alone in the treatment of HER2-positive metastatic breast cancer is in progress and the results will be released in the near future. If the results indicate a superior effect of paclitaxel plus lapatinib combination compared to paclitaxel alone, lapatinib will become another first-line target therapy drug after trastuzumab in the treatment of HER2-positive metastatic breast cancer.

Although both lapatinib and trastuzumab are important target therapeutic drugs for HER2-positive breast cancer, they have different therapeutic mechanisms and possibly incomplete cross-resistance. The intracellular mechanism of action of lapatinib provides the rationale for evaluating lapatinib in trastuzumab-resistant disease. Preclinical studies demonstrated that lapatinib induced apoptosis and inhibited insulin-like growth factor I receptor signaling in trastuzumab-resistant breast cancer cell lines, and inhibited phosphorylation of the constitutively activated, truncated HER2 receptor p95ErbB2. ${ }^{10,53-55}$ Both phosphatase and tensin homolog (PTEN) deficiency and increased signaling through p95ErbB2-containing heterodimers, which do not contain the HER2 extracellular domain to which trastuzumab binds, have been proposed as mechanisms of trastuzumab resistance. ${ }^{56} \mathrm{Ma}$ et $\mathrm{al}^{57}$ studied 35 metastatic breast cancer patients who had disease progression after a lapatinib-based combination therapy (the patients had never been treated with trastuzumab before). The patients were divided into two groups: control group $(n=22)$ who received chemotherapy or endocrine therapy alone and trastuzumab group ( $n=13$ ) who received trastuzumab-based combination therapy. The two groups were similar in age, estrogen and progesterone receptor status, location of metastasis, number of metastasis, disease-free survival, and progression-free survival. In the control group, 59\% of patients received chemotherapy and the remaining $41 \%$ of patients received endocrine therapy. In the trastuzumab group, $69 \%$ of patients received combination with chemotherapy and others with endocrine therapy. The median follow-up period was 58 months. The trastuzumab group and the control group had median progression-free survival of 10.0 months and 3.3 months $(P=0.001)$, and total survival of 31.1 months and 7.0 months $(P=0.015)$, respectively. These data suggest that trastuzumab is effective in the treatment of lapatinib-resistant breast cancer. Moreover, lapatinib retained significant growth inhibitory activity against HER2-overexpressing breast cancer cell lines selected for long-term ( $>9$ months) outgrowth in trastuzumab-containing culture medium, thus suggesting a lack of cross-resistance between these two HER2-directed agents. ${ }^{10}$

Approximately, one third of advanced HER2-positive breast cancer patients develop brain metastases. Treatment 
options are extremely limited for CNS progression after intracranial radiation therapy and brain metastases have become one of the greatest challenges in clinical management. Recent studies have shown that lapatinib can more easily traverse the blood-brain barrier and may have an advantage compared to trastuzumab in the prevention and/ or treatment of CNS metastases. ${ }^{21,23,58}$ Thus, lapatinib is considered as a novel targeted therapy for breast cancer brain metastasis. Lin et al ${ }^{59}$ collected 242 cases with advanced breast cancer associated with CNS metastasis in the study. Of the 50 evaluable patients who received lapatinib-based combination therapy, $20 \%$ experienced a CNS objective response and $40 \%$ experienced $\geq 20 \%$ volumetric reduction in their CNS lesions. This study confirms the modest CNS antitumor activity of lapatinib. Additional responses were observed with the combination of lapatinib and capecitabine. Further studies of lapatinib-based regimens for CNS metastases from HER2-positive breast cancer are warranted.

Although lapatinib is effective for metastatic breast cancer treatment, it has not been approved by health insurance companies, thus limiting its clinical application. It has only been tried in some patients with disease progression after chemotherapy and trastuzumab therapy in the People's Republic of China.

\section{Conclusion}

Lapatinib is an orally active small molecule inhibitor of the intracellular tyrosine kinase domains of EGFR and HER2. A combination of lapatinib and capecitabine provided an effective and well-tolerated treatment option in a population of Chinese women with HER2-positive advanced or metastatic breast cancer who who have had disease progression following other treatments. The clinical benefit rate of lapatinib in the Chinese population is considerably higher than that in a Western population with a favorable median progression-free survival. Additional studies of lapatinib plus capecitabine for brain metastases from HER2-positive breast cancer are warranted.

\section{Disclosure}

The authors report no conflicts of interest in this work.

\section{References}

1. Jemal A, Bray F, Center MM, et al. Global cancer statistics. CA Cancer J Clin. 2011;61(2):69-90.

2. Wolff AC, Hammond ME, Schwartz JN, et al. American Society of Clinical Oncology/College of American Pathologists guideline recommendations for human epidermal growth factor receptor 2 testing in breast cancer. Arch Pathol Lab Med. 2007;131(1):18-43.
3. Ross JS, Slodkowska EA, Symmans WF, et al. The HER-2 receptor and breast cancer: ten years of targeted anti-HER-2 therapy and personalized medicine. Oncologist. 2009;14(4):320-368.

4. Rasmussen BB, Regan MM, Lykkesfeldt AE, et al. Adjuvant letrozole versus tamoxifen according to centrally-assessed ERBB2 status for postmenopausal women with endocrine-responsive early breast cancer: supplementary results from the BIG 1-98 randomised trial. Lancet Oncol. 2008;9(1):23-28.

5. Tripathy D, Slamon DJ, Cobleigh M, et al. Safety of treatment of metastatic breast cancer with trastuzumab beyond disease progression. J Clin Oncol. 2004;22(6):1063-1070.

6. Montemurro F, Donadio M, Clavarezza M, et al. Outcome of patients with HER2-positive advanced breast cancer progressing during trastuzumab-based therapy. Oncologist. 2006;11(4):318-324.

7. Cameron D. Lapatinib plus capecitabine in patients with HER2positive advanced breast cancer. Clin Adv Hematol Oncol. 2007;5(6): 456-458.

8. Spector NL, Xia W, Burris H 3rd, et al. Study of the biologic effects of lapatinib, a reversible inhibitor of ErbB1 and ErbB2 tyrosine kinases, on tumor growth and survival pathways in patients with advanced malignancies. J Clin Oncol. 2005;23(11):2502-2512.

9. Xia W, Mullin RJ, Keith BR, et al. Anti-tumor activity of GW572016: a dual tyrosine kinase inhibitor blocks EGF activation of EGFR/erbB2 and downstream Erk1/2 and AKT pathways. Oncogene. 2002;21(41): 6255-6263.

10. Konecny GE, Pegram MD, Venkatesan N, et al. Activity of the dual kinase inhibitor lapatinib (GW572016) against HER-2-overexpressing and trastuzumab-treated breast cancer cells. Cancer Res. 2006;66(3): 1630-1639.

11. Polier S, Samant RS, Clarke PA, et al. ATP-competitive inhibitors block protein kinase recruitment to the Hsp90-Cdc37 system. Nat Chem Biol. 2013;9(5):307-312.

12. Rexer BN, Ham AJ, Rinehart C, et al. Phosphoproteomic mass spectrometry profiling links Src family kinases to escape from HER2 tyrosine kinase inhibition. Oncogene. 2011;30(40):4163-4174.

13. Lee YY, Kim HP, Kang MJ, et al. Phosphoproteomic analysis identifies activated MET-axis PI3K/AKT and MAPK/ERK in lapatinib-resistant cancer cell line. Exp Mol Med. 2013;45:e64.

14. Rusnak DW, Lackey K, Affleck K, et al. The effects of the novel, reversible epidermal growth factor receptor/ErbB-2 tyrosine kinase inhibitor, GW2016, on the growth of human normal and tumor-derived cell lines in vitro and in vivo. Mol Cancer Ther. 2001;1(2):85-94.

15. Nelson MH, Dolder CR. Lapatinib: a novel dual tyrosine kinase inhibitor with activity in solid tumors. Ann Pharmacother. 2006;40(2): 261-269.

16. Satoh T, Xu RH, Chung HC, et al. Lapatinib Plus Paclitaxel Versus Paclitaxel Alone in the Second-Line Treatment of HER2-Amplified Advanced Gastric Cancer in Asian Populations: TyTAN - A Randomized, Phase III Study. J Clin Oncol. 2014;32(19):2039-2049.

17. Gandhi MD, Agulnik M. Targeted treatment of head and neck squamous-cell carcinoma: potential of lapatinib. Onco Targets Ther. 2014;7:245-251

18. Polli JW, Humphreys JE, Harmon KA, et al. The role of efflux and uptake transporters in [N-\{3-chloro-4-[(3-fluorobenzyl)oxy]phenyl $\}-6-[5-(\{[2-$ (methylsulfonyl)ethyl]amino methyl)-2-furyl]-4-quinazolinamine (GW572016, lapatinib) disposition and drug interactions. Drug Metab Dispos. 2008;36(4):695-701.

19. Polli JW, Olson KL, Chism JP, et al. An unexpected synergist role of P-glycoprotein and breast cancer resistance protein on the central nervous system penetration of the tyrosine kinase inhibitor lapatinib (N-\{3-chloro-4-[(3-fluorobenzyl)oxy]phenyl \}6-[5-(\{[2-(methylsulfonyl)ethyl]amino $\}$ methyl)-2-furyl $]-4$ quinazolinamine; GW572016). Drug Metab Dispos. 2009;37(2): 439-442.

20. Gerstner ER, Fine RL. Increased permeability of the blood-brain barrier to chemotherapy in metastatic brain tumors: establishing a treatment paradigm. J Clin Oncol. 2007;25(16):2306-2312. 
21. Steeg PS, Camphausen KA, Smith QR. Brain metastases as preventive and therapeutic targets. Nat Rev Cancer. 2011;11(5): 352-363.

22. Taskar KS, Rudraraju V, Mittapalli RK, et al. Lapatinib distribution in HER2 overexpressing experimental brain metastases of breast cancer. Pharm Res. 2012;29(3):770-781.

23. Gril B, Palmieri D, Bronder JL, et al. Effect of lapatinib on the outgrowth of metastatic breast cancer cells to the brain. J Natl Cancer Inst. 2008;100(15):1092-1103.

24. Kaplan MA, Ertugrul H, Firat U, et al. Brain metastases in HER2positive metastatic breast cancer patients who received chemotherapy with or without trastuzumab. Breast Cancer. Epub January 3, 2014.

25. Lin NU, Freedman RA, Ramakrishna N, et al. A phase I study of lapatinib with whole brain radiotherapy in patients with Human Epidermal Growth Factor Receptor 2 (HER2)-positive breast cancer brain metastases. Breast Cancer Res Treat. 2013;142(2):405-414.

26. Wood ER, Truesdale AT, McDonald OB, et al. A unique structure for epidermal growth factor receptor bound to GW572016 (Lapatinib): relationships among protein conformation, inhibitor off-rate, and receptor activity in tumor cells. Cancer Res. 2004;64(18):6652-6659.

27. Rusnak DW, Affleck K, Cockerill SG, et al. The characterization of novel, dual ErbB-2/EGFR, tyrosine kinase inhibitors: potential therapy for cancer. Cancer Res. 2001;61(19):7196-7203.

28. Force T, Kolaja KL. Cardiotoxicity of kinase inhibitors: the prediction and translation of preclinical models to clinical outcomes. Nat Rev Drug Discov. 2011;10(2):111-126.

29. Fedele C, Riccio G, Coppola C, et al. Comparison of preclinical cardiotoxic effects of different ErbB2 inhibitors. Breast Cancer Res Treat. 2012;133(2):511-521.

30. FDA. Tykerb (Lapatinib) FDA NDA Pharmacology Review [webpage on the Internet]. 2008:Available from: http://www.accessdata.fda.gov/ drugsatfda_docs/nda/2007/022059s000TOC.cfm. Accessed on July 16, 2014.

31. Dogan E, Yorgun H, Petekkaya I, et al. Evaluation of cardiac safety of lapatinib therapy for ErbB2-positive metastatic breast cancer: a single center experience. Med Oncol. 2012;29(5):3232-3239.

32. Spector NL, Yarden Y, Smith B, et al. Activation of AMP-activated protein kinase by human EGF receptor 2/EGF receptor tyrosine kinase inhibitor protects cardiac cells. Proc Natl Acad Sci U SA. 2007;104(25): 10607-10612.

33. Tykerb ${ }^{\circledR}$ [package insert]. Research Triangle Park, NC: GlaxoSmithKline; 2007.

34. Chu I, Blackwell K, Chen S, Slingerland J. The dual ErbB1/ErbB2 inhibitor, lapatinib (GW572016), cooperates with tamoxifen to inhibit both cell proliferation- and estrogen-dependent gene expression in antiestrogen-resistant breast cancer. Cancer Res. 2005;65(1): $18-25$.

35. Scaltriti M, Verma C, Guzman M, et al. Lapatinib, a HER2 tyrosine kinase inhibitor, induces stabilization and accumulation of HER2 and potentiates trastuzumab-dependent cell cytotoxicity. Oncogene. 2009;28(6):803-814.

36. Li Z, Chang T, Shi 1. E R a 通路代偿性激活在乳腺癌细胞的拉 帕替尼获得性耐药中的作用 [Compensatory activation of estrogen receptor alpha signaling in acquired resistance to lapatinib of HER-2 overexpressing/ER alpha-positive breast cancer cells]. Academic Journal of Second Military Medical University. 2013;34:616-622. Chinese.

37. Zhao L. Wnt/ $\beta$-catenin信号通路在乳腺癌拉帕替尼耐药中的作 用 [Effect of Wnt/ $\beta$-catenin pathway on lapatinib resistance in breast cancer]. Jiangsu Medical Journal. 2013;39:2242-2249. Chinese.

38. Zhang H, Mao X, Lin Z, et al. 拉帕替尼诱导Her-2 高表达乳腺癌细 胞调亡的机制探讨 [Mechanism of Her-2 overexpressed breast cancer cell apoptosis induced by lapatinib]. Shandong Medicine Journal. 2011;51:1-3. Chinese.

39. Schluter B, Gerhards R, Strumberg D, Voigtmann R. Combined detection of Her2/neu gene amplification and protein overexpression in effusions from patients with breast and ovarian cancer. J Cancer Res Clin Oncol. 2010;136(9):1389-1400.
40. Geyer CE, Forster J, Lindquist D, et al. Lapatinib plus capecitabine for HER2-positive advanced breast cancer. N Engl J Med. 2006;355(26): 2733-2743.

41. Goss PE, Smith IE, O'Shaughnessy J, et al. Adjuvant lapatinib for women with early-stage HER2-positive breast cancer: a randomised, controlled, phase 3 trial. Lancet Oncol. 2013;14(1):88-96.

42. Chong HY, Taib NA, Rampal S, et al. Treatment options for locally advanced breast cancer - experience in an Asian tertiary hospital. Asian Pac J Cancer Prev. 2010;11(4):913-917.

43. Lal S, Wong ZW, Jada SR, et al. Novel SLC22A16 polymorphisms and influence on doxorubicin pharmacokinetics in Asian breast cancer patients. Pharmacogenomics. 2007;8(6):567-575.

44. Han C, You J, Jing F, et al. 拉帕替尼治疗HER-2阳性晚期乳腺癌的 疗效观察 [Therapeutic effect of lapatinib on HER-2 positive advanced breast cancer]. J Chinese PLA Postgrad Med Sch. 2013;4:325-326. Chinese.

45. Xu BH, Jiang ZF, Chua D, et al. Lapatinib plus capecitabine in treating HER2-positive advanced breast cancer: efficacy, safety, and biomarker results from Chinese patients. Chin J Cancer. 2011;30(5):327-335.

46. Cameron D, Casey M, Press M, et al. A phase III randomized comparison of lapatinib plus capecitabine versus capecitabine alone in women with advanced breast cancer that has progressed on trastuzumab: updated efficacy and biomarker analyses. Breast Cancer Res Treat. 2008;112(3):533-543.

47. Shepherd FA, Rodrigues Pereira J, Ciuleanu T, et al. Erlotinib in previously treated non-small-cell lung cancer. $N$ Engl J Med. 2005;353(2): 123-132.

48. Mok TS, Wu YL, Thongprasert S, et al. Gefitinib or carboplatinpaclitaxel in pulmonary adenocarcinoma. N Engl J Med. 2009;361(10): 947-957.

49. Xu B. 晚期乳腺癌的治疗原则与策略 [Therapeutic criteria and strategy in advanced breast cancer]. China Medical Herald. 2010;7:6-8. Chinese.

50. Jiang Z, Shao Z, Xu B. 人表皮生长因子受体2阳性乳腺癌临床诊 疗专家共识 [National guidline for management of human epidermal growth factor receptor 2 positive breast cancer]. Chin J Oncol. 2010;32:158-160. Chinese.

51. Zhou X, Cella D, Cameron D, et al. Lapatinib plus capecitabine versus capecitabine alone for HER2+ (ErbB2+) metastatic breast cancer: quality-of-life assessment. Breast Cancer Res Treat. 2009;117(3): 577-589.

52. Wiechmann L, Sampson M, Stempel M, et al. Presenting features of breast cancer differ by molecular subtype. Ann Surg Oncol. 2009;16(10): 2705-2710.

53. Xia W, Liu LH, Ho P, Spector NL. Truncated ErbB2 receptor (p95ErbB2) is regulated by heregulin through heterodimer formation with ErbB3 yet remains sensitive to the dual EGFR/ErbB2 kinase inhibitor GW572016. Oncogene. 2004;23(3):646-653.

54. Nahta R, Yuan LX, Du Y, Esteva FJ. Lapatinib induces apoptosis in trastuzumab-resistant breast cancer cells: effects on insulin-like growth factor I signaling. Mol Cancer Ther. 2007;6(2):667-674.

55. Xia W, Husain I, Liu L, et al. Lapatinib antitumor activity is not dependent upon phosphatase and tensin homologue deleted on chromosome 10 in ErbB2-overexpressing breast cancers. Cancer Res. 2007;67(3): 1170-1175.

56. Nahta R, Yu D, Hung MC, Hortobagyi GN, Esteva FJ. Mechanisms of disease: understanding resistance to HER2-targeted therapy in human breast cancer. Nat Clin Pract Oncol. 2006;3(5):269-280.

57. $\mathrm{Ma} \mathrm{F}, \mathrm{Li} \mathrm{Q}, \mathrm{Li} \mathrm{H}$, et al. 曲妥珠单抗治疗拉帕替尼耐药乳腺癌的临床 价值 [Clinical evaluation of trastuzumab in the treatment of lapatinibresistant breast cancer] Zhong Hua Yi Xue Zhong Liu Xue Hui Lun Wen Hui Bian. 2011;7:6-8. Chinese.

58. Moy B, Goss PE. Lapatinib: current status and future directions in breast cancer. Oncologist. 2006;11(10):1047-1057.

59. Lin NU, Dieras V, Paul D, et al. Multicenter phase II study of lapatinib in patients with brain metastases from HER2-positive breast cancer. Clin Cancer Res. 2009;15(4):1452-1459. 


\section{Publish your work in this journal}

OncoTargets and Therapy is an international, peer-reviewed, open access journal focusing on the pathological basis of all cancers, potential targets for therapy and treatment protocols employed to improve the management of cancer patients. The journal also focuses on the impact of management programs and new therapeutic agents and protocols on
Dovepress

patient perspectives such as quality of life, adherence and satisfaction. The manuscript management system is completely online and includes a very quick and fair peer-review system, which is all easy to use. Visit http://www.dovepress.com/testimonials.php to read real quotes from published authors.

\footnotetext{
Submit your manuscript here: http://www.dovepress.com/oncotargets-and-therapy-journal
} 